\title{
Calculation of the heat power consumption in the heat exchanger using artificial neural network
}

by S. Dudzik*

\author{
* Faculty of Electrical Engineering, Institute of Electronics and Control Systems - Częstochowa University of \\ Technology, Częstochowa, Poland
}

\section{Abstract}

In this paper a new method for calculation of heat power consumption in a heat exchanger is described. The method is based on the analysis of phenomena occurring between the heat exchanger and the ambient. The calculation of heat power consumption represents an inverse heat transfer problem [1, 2]. In this work the experimental and simulation investigations were conducted. An artificial neural network, trained with data obtained from infrared thermography measurements [3-6] is used to calculate the heat power consumption in steady state. In this work the results of simulations under the assumption of different variants of input data selection were presented. The experiments were carried out using measuring stand with open chamber [7].

\section{Introduction}

The solution of coefficient inverse heat conduction problems was presented e.g. in [8, 9]. In this paper, an artificial neural network is used to calculate the heat power consumption of a radiator (e.g. in a central heating system). The input data for training networks were obtained from differential thermograms of considered radiator surface. These thermograms were created on the basis of the thermal images acquired with an infrared camera, and the measurements of the ambient temperature. The output data (measured heat power consumption) were obtained using the measuring stand with an open chamber equipped with a PC and suitable measurement devices.

In this work it was assumed, that the heat from the radiator is emitted by convection and radiation. The convective component of the heat flux can be approximated with the following formula:

$$
\dot{Q}=\alpha\left(\bar{T}_{R}-T_{a m b}\right) \text {, }
$$

where: $\bar{T}_{R}, \mathrm{~K}$ - the average temperature of considered heat exchanger, $T_{a m b}, \mathrm{~K}-$ the ambient temperature, measured in the reference point. In order to determine the $\alpha$ coefficient, the similarity numbers like the Nusselt, Prandtl and Grasfoff number can be used. For example, the Nusselt number is equal to:

$$
N u=c(R a)^{w}=\frac{\alpha d}{\lambda},
$$

where: $c$-constant, $d$-characteristic dimension, $\lambda$ - thermal conductivity, $R a$ - Rayleigh number, $w-$ exponent of about from 0,25 to 0,33 . On the basis of empirical experiments the following formula describing of $\alpha$ coefficient for a vertical isothermal plate was derived [10]:

$$
\alpha=1,62\left(\bar{T}_{R}-T_{\text {amb }}\right)^{0,25}+0,128\left(\bar{T}_{R}-T_{0}\right)^{0,54}
$$

Since the $\alpha$ coefficient is described as above, the convective heat flux referred to the unit surface is defined as:

$$
\frac{d \dot{Q}_{k}}{d F}=\left[1,62+0,128\left(\bar{T}_{R}-T_{a m b}\right)^{0,29}\right]\left(\bar{T}_{R}-T_{a m b}\right)^{1,25}
$$

The radiation component of the heat flux referred to the unit surface, under the simplifying assumptions is described with the following equation:

$$
\left(\frac{d \dot{Q}_{r}}{d F}\right)=\varepsilon_{R} \phi \sigma\left(T_{R}^{4}-T_{\mathrm{amb}}{ }^{4}\right),
$$

where: $\varepsilon_{R}$ - emissivity of the radiator surface, $\phi$ - view factor of the radiator surface, $\sigma-$ StefanBoltzmann constant.

In the approximated equations introduced above, the heat power consumption depends on the difference between the average temperature of the radiator surface and the ambient temperature. Unfortunately, in practice, the average temperature of the radiator surface is unknown. In most cases, the place where the average temperature occurs is unknown as well. For this reason in this work the temperature distribution on the front surface of the considered radiator was used. Additionally, because the equations $(4,5)$ are valid only for simplified heat transfer conditions (including geometry of radiator) in order to determine the amount of heat consumption the neural network model was used. 


\section{Experimental research}

\section{Measuring position}

In the experiments, a panel radiator was used. In order to obtain the training and testing data, the measuring stand was used. Measurements were conducted using the open chamber. The experimental setup is shown in Fig. 1. The following denotations are assumed in Fig. 1: 1-the heater under investigation, $2-$ the feed water heater, 3 - the membrane expansion vessel, 4 - the circulating pump, 5 - the flow regulating valves, 6 the heater valves, 7 - the vent valve, 8 - the industrial temperature controller, 9 - the solid state relay, 10 - the heat meter type of MULTICAL 66C with RS232 serial interface, 11 - the points of the temperature measurement, 12 - the ultrasonic flow transducer, 13 - the strip with the ambient temperature sensors located along the height of the chamber, 14 - the data acquisition card Ni USB 6008, 15 - PC with the data acquisition software created in the LabVIEW environment.

In the experiments the following steps were assumed:

1. Determination of the standard characteristic of considered radiator on the stand with the closed chamber.

2. Determination of the heat power consumption on the position with the open chamber, using the standard characteristic, and additionally determination of the distribution of the temperature on the front surface of the considered radiator with infrared camera.

The measurements in the closed chamber were conducted in accordance with EN 442-2:1999 standard. In order to obtain the measuring data, the open chamber was used. The measuring stand was equipped with the PC computer with the LabVIEW software. Additionally, the computer was equipped in the data acquisition card. In order to measure the water parameters (e. g. the inlet $\left(t_{1}\right)$ and outlet $\left(t_{2}\right)$ temperatures, volumetric flow $\left(q_{v}\right)$ of the water) the intelligent heat meter with the RS232 serial interface was used. In this work, in order to control the data acquisition process, a program in LabVIEW was created. The sampled values of measured quantities were stored and then they were processed in the MATLAB computational environment. It was possible to calculate the heat power consumptions for the different conditions of the water and ambient. Additionally, on the measuring stand, the distribution of the temperature was obtained. It was done with FLIR ThermaCAM PM595 infrared camera.

The exemplary results of the experimental investigations are shown in Fig. 2, 3. In these figures the thermograms of the frontal surface of the radiator for the different measuring conditions were presented.

\section{The variants of the input data selection}

The process of obtaining the data for the artificial neural network can be conducted in the following steps:

Recording of the thermograms of the investigated radiator on the measured stand with the open chamber in a steady state.

Averaging of the thermogram series and making of the differential thermogram.

Making of the input data sets according to assumed variant of the input data selection with the specialized software (on the basis of the differential thermogram).

In the considered heat transfer process, the most important role plays the temperature difference occuring between the radiator surface and the ambient [2, 11]. Furthermore, in the non-isothermal surface case, the temperature distribution of this surface is required. In this paper the differential thermogram was used as the input data source. During the experimental research, the series of 60 thermograms of the front surface of the radiator was recorded. Then, the averaging of all the thermograms for each pixels was made. The averaging was made using the following formula:

$$
\bar{t}(x, y)=\frac{1}{60} \sum_{i=1}^{60} t_{i}(x, y),
$$

where: $\bar{t}(x, y)$ - averaged temperature for the $(x, y)$ pixel coordinates in the thermogram plane, ${ }^{\circ} \mathrm{C} ; t_{i}(x, y)$ - temperature of $i$-th pixel value in the thermogram plane, ${ }^{\circ} \mathrm{C}$.

Besides of the recording of the temperature distribution, the reference ambient temperature was measured as well. The reference ambient temperature denotes the air temperature in the central axis of the exploratory chamber, across from the investigated radiator, on the height of $(0,75 \pm 0,001) \mathrm{m}$ above the floor, in accordance with the recommendation of the DIN 4704-2 standard. The differential thermogram was made by subtracting the reference ambient temperature from each of the thermogram pixels. It was done by following formula:

$t_{\Delta}(x, y)=\bar{t}(x, y)-t_{r},(7)$

where: $t_{\Delta}(x, y)$ - the temperature for the $(x, y)$ pixel coordinates in the differential thermogram plane, ${ }^{\circ} \mathrm{C}$;

$t_{r}$ - the reference ambient temperature, ${ }^{\circ} \mathrm{C}$.

The differential thermograms for different values of the ambient temperature and water parameters are presented in the fig. 4, 5. Next, the differential control areas on the frontal surface of the radiator were assumed. In this work the control area locations were called the variants of input data selection. During the simulations of the algorithm for calculation of the heat power consumption, the criteria of the input data selection were assumed. For definition of the input data selection variants, the followed assumptions were made: 
Accessibility of the function carrying out the chosen parameter determination (e. g. average temperature for the area, temperature profiles) in the infrared system.

Possibility of application of the described algorithm in the measurement systems without infrared camera (e. g. temperature measurement made using contact methods)

In this paper the results obtaining from two variants of input data selection were compared. The following variants were examined:

The difference between the averaged temperature from one control area (in the form of the square about $10 \times 10$ pixels) and the reference ambient temperature - single-input neural network - Fig. 6 .

The difference between the averaged temperature from the three control areas (in the form of the square $10 \times 10$ pixels) and the reference ambient temperature - three-input neural network - Fig. 7.

\section{Simulation research}

\section{Algorithm for calculation of heat power consumption}

The solutions of coefficient inverse heat conduction problems using artificial neural networks were presented e. g. in $[8,9,12,13]$. In this paper, an artificial feed-forward neural network and backpropagation algorithm were used to calculate the heat power consumption of a radiator in a central heating system. The input data for training the network were obtained from thermograms of the considered radiator surface and the ambient temperature, measured at the reference point. The output data (the measured heat power consumption) were obtained using the measuring stand with open chamber equipped with a PC and suitable measurement instruments [7]. The proposed algorithm for calculation of heat power consumption operates in the two following modes:

The training mode. In this mode the selection of the neural network weights is made. The data obtained from thermovision and ambient temperature measurements are applied to the net inputs, according to the suitable variant of input data selection. The measured heat power consumption is applied to the output of the neural network. It is measured with the measuring stand or calculated using a numerical model.

The reconstruction mode. In this mode, the on-line recorded values are applied to the inputs of the net. On the output the calculated value of the heat power consumption value is obtained.

The neural network, used in the described algorithm is shown in Fig. 8, where: $x_{1}, x_{2}, x_{3}-$ inputs of the neural network (e. g. averaged temperature values of control areas); $f^{1}$ - activation function of neurons in the hidden layer (linear or tangent curve); $f^{2}$ - activation function of neurons in the output layer (as above), $b^{1}{ }_{K}-$ biases of neurons in the hidden layer, $b^{2}-$ bias of neuron in the output layer. $\Phi_{N E T}$ - measured power consumption in investigated heat exchanger.

1. The simulations of the described algorithm were carried out in following stages:

2. Division of data set into the training, testing and validating data sets.

3. Normalization of data sets.

4. Training of networks using different optimization algorithms and different network architectures.

5. Simulation of neural networks, in particular for testing data.

6. Analysis of simulation results.

\section{The networks architectures used in the simulations}

One of the most important properties of neural networks in the approximation problems is the generalization ability [2]. In the training the presented samples come from training data set. However, the ultimate criterion of the approximation quality is an error on the testing data set (the testing data does not participate in the training). Therefore, the most important problem is the suitable selection of the training data samples and the number of neurons in the hidden layer of the considered neural network.

Taking into consideration the above conditions, in the simulation research of the described algorithm, it was necessary to divide the measured data into three subsets: training, validating and testing. The simulations were conducted with the assumption of uniformly-spaced data samples.

Table 1. Grouping of the data used in the simulations

\begin{tabular}{|c|c|}
\hline Set of data & $\begin{array}{c}\text { Number of } \\
\text { data samples }\end{array}$ \\
\hline Training & $80 \%$ \\
\hline Testing & $10 \%$ \\
\hline Validating & $10 \%$ \\
\hline
\end{tabular}

The fundamental problem is the appropriate selection of the network architecture. In the paper the feedforward architecture is assumed. The two-layer network was also assumed. The results presented in the paper were obtained, taking into account that the activation functions of the neurons in the hidden layer are sigmoid and the neurons in the output layer are linear. 


\section{Results of the simulation research}

In order to determine the approximation quality, the following criteria were computed for each of networks: The relative error of the algorithm for a single sample:

$\delta_{N E T}=\frac{\Phi_{N E T}-\Phi_{M E S}}{\Phi_{M E S}}, \%$,

where: $\Phi_{N E T}$ - value of the heat power consumption, on the output of the network, W; $\Phi_{M E S}$ value of the heat power consumption measured in the experimental research, $\mathrm{W}$.

The limiting relative error of the algorithm for all the measured data:

$\widehat{\delta}_{N E T}=\operatorname{MAX}\left\{\delta_{N E T}\right\}, \%$

The limiting error of the algorithm within the range of the standard characteristic:

$\hat{\delta}_{N E T}^{\text {norm }}=\operatorname{MAX}\left\{\delta_{N E T}^{\text {norm }}\right\}, \%$,

where: $\delta_{N E T}^{n o r m}$ - relative error for each sample within the range of the standard characteristic.

The results of the simulations research are presented in Fig. 10-12. The relative error of the algorithm for 2 and 10 neurons in the hidden layer for the first variant of input data selection are depicted in Fig. 9, 10 and for the second variant in fig. 11, 12.

\section{Conclusions}

The application of the described algorithm, trained with thermovision data, allows for the calculation of heat power consumption in steady state.

A very useful input data source for the described algorithm is the differential thermogram.

Use of neural network models, trained with thermovision data, gives the limiting error less or equal $2 \%$, if the measured heat power consumption is contained in the range of the normal characteristic.

The value of the limiting error depends on the input data selection. The better results were obtained for the second variant of the input data selection (three-input neural network). algorithm.

The best simulation results were obtained for networks trained with Levenberg-Marquardt optimization The presented algorithm for calculation of the heat power consumption can be considered as a prototype of noncontact heat measurement system working in the steady state.

\section{REFERENCES}

[1] Beck J. V., Blackwell B., Clair JR, Ch. R. St.: „Inverse heat conduction”, John Wiley \& Sons Inc., New York 1985.

[2] Kreith F.: „The CRC Handbook of Thermal Engineering”, CRC Press LLC, 2000.

[3] Minkina W., Dudzik S.: „Simulation analysis of uncertainty of infrared camera measurement and processing path", Measurement - Vol. 39, Issue 8, pp 758-763, Elsevier Ltd, 2006.

[4] Astarita T., Cardone G., Carlomagno G.M., Meola C.: „A survey on infrared thermography for convective heat transfer measurements", Optics \& Laser Technology, Vol. 32 (2000) pp. 593-610, Elsevier Science Ltd, 2000.

[5] Minkina W.: "Thermovision Measurements - Methods and Instruments", Technical University of Czestochowa Publishers, Czestochowa 2004, ISBN 83-7193-237-5.

[6] Babka R., Minkina W.: „Sub-pixel edge location in thermal images using a meansquare measure" 7th International Conference on Infrared Sensors \& Systems (IRS2'2002), 1416.05.2002, Erfurt, RFN, str. 213-218.

[7] DIN 4704-2: „Prüfung von Raumheizkörpern, Offene Prüfkabine”, 1998.

[8] Islamoglu Y.: „A new approach for the prediction of the heat transfer rate of the wire-on-tube type heat exchanger-use of an artificial neural network model", Applied Thermal Engineering Vol. 23 (2003), pp. 243-249, Elsevier Science Ltd, 2003.

[9] Raudensky M., Horsky J., Krejsa J.: „Usage of neural network for copled parameter and function specification inverse heat conduction problem" International Communications in Heat Mass Transfer Vol. 22, No. 5 pp. 661-670, Elsevier Science Ltd. 1995.

[10] Adunka F.: „Wärmemengenmessung”, Vulkan-Verlag, Essen, 1984.

[11] Wark K. Jr.: "Thermodynamics”, McGraw-Hill Book Company, New York 1998.

[12] Minkina W., Chudzik S.: "The measurements of the thermal parameters of the heat insulating materials Instruments and methods", Technical University of Czestochowa Publishers, Częstochowa 2004, ISBN 837193-216-2 (in polish).

[13] Dudzik S.: "The application of the artificial neural network trained with thermovision data to the approximation of the plate heat exchanger in the condition of the natural convection", Proceedings of the Central European VI Conference Scientific-Engineering: „Computer-aided Methods and Systems in the Automatics and Electrical Engineering”, Częstochowa-Poraj, 16-17.09.2005r., pp. 52-57 (in polish). 


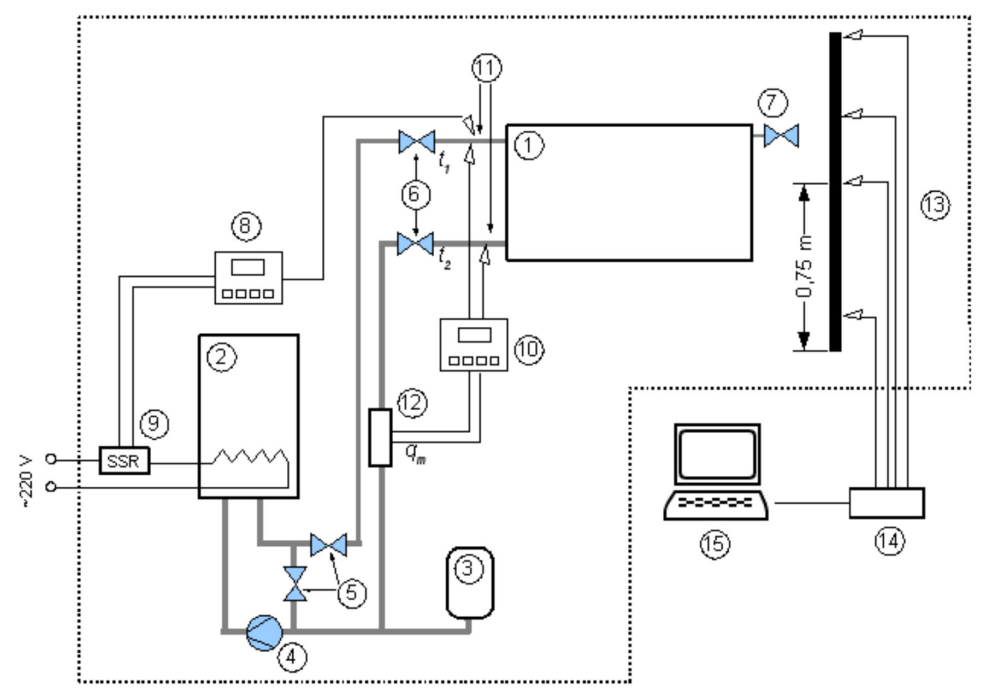

Fig. 1. Experimental setup used in the experimental research

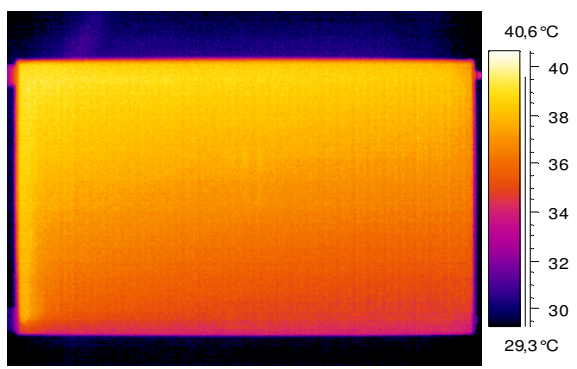

Fig. 2. Thermogram of the frontal surface of the investigated heater $\left(t_{1}=40^{\circ} \mathrm{C}, q_{v}=30 \mathrm{l} / \mathrm{h}\right)$

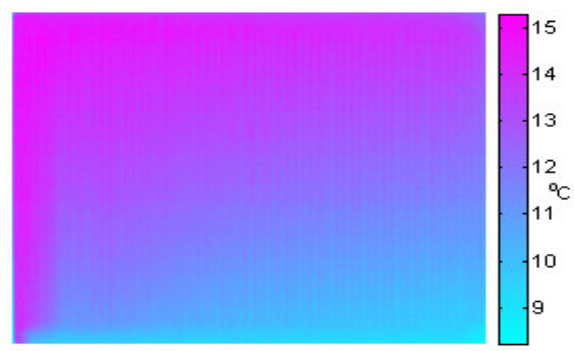

Fig. 4. Differential thermogram of the frontal surface of the investigated heater $\left(t_{1}=40\right.$ $\left.{ }^{\circ} \mathrm{C}, q_{v}=30 \mathrm{l} / \mathrm{h}\right)$

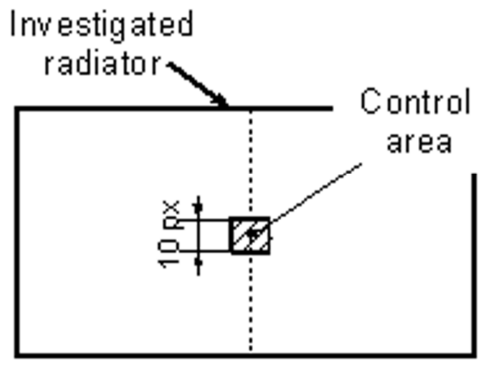

Fig. 6. Locations of the control area in the first variant of input data selection

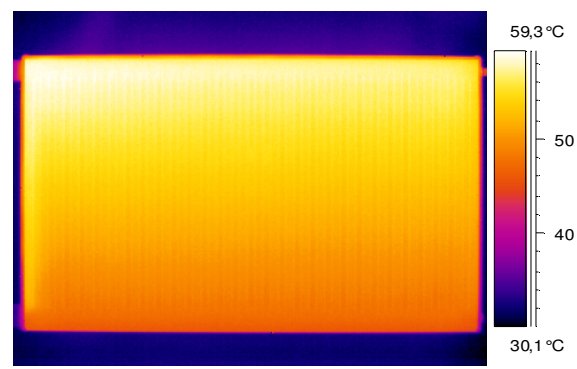

Fig. 3. Thermogram of the frontal surface of the investigated heater $\left(t_{1}=60^{\circ} \mathrm{C}, q_{v}=30 \mathrm{l} / \mathrm{h}\right)$

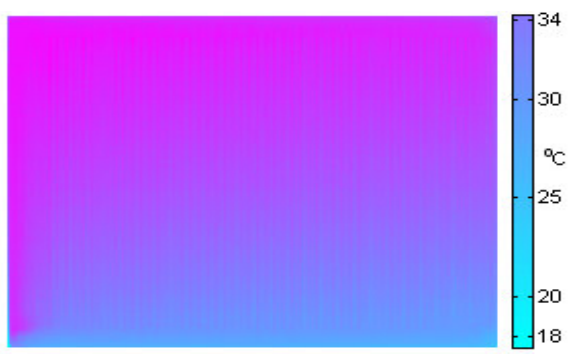

Fig. 5. Differential thermogram of the frontal surface of the investigated heater $\left(t_{1}=60\right.$ ${ }^{\circ} \mathrm{C}, q_{v}=30 \mathrm{l} / \mathrm{h}$ )

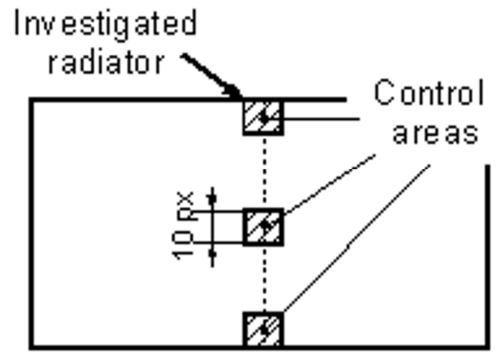

Fig. 7. Locations of the control area in the second variant of input data selection 


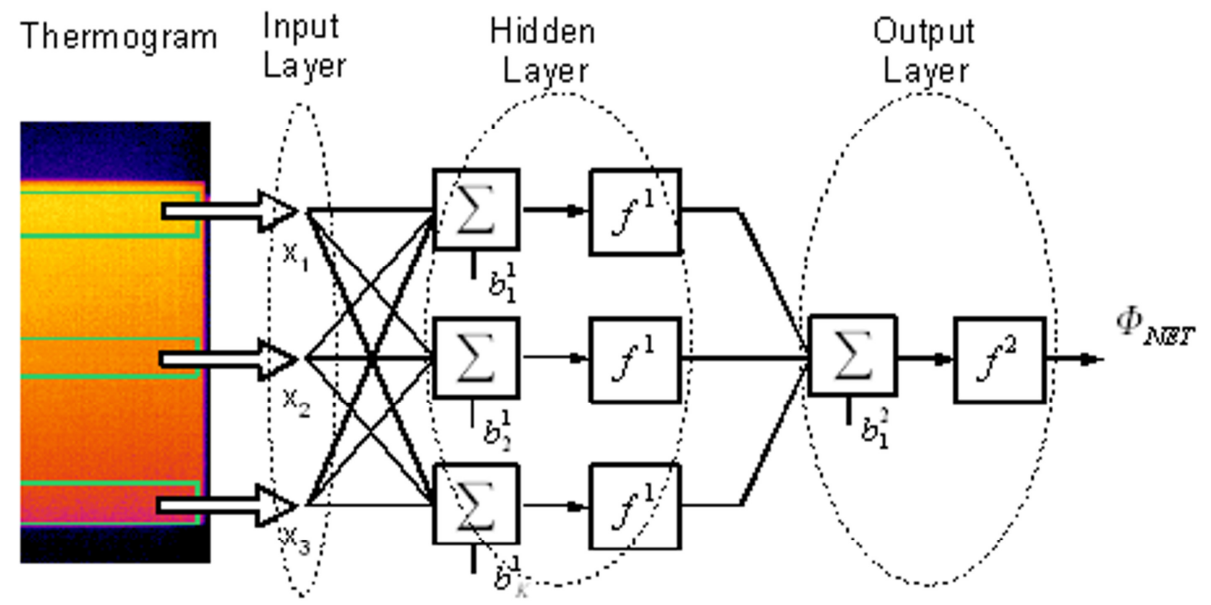

$x_{1}$ - statistical parameters of the control areas

Fig. 8. Diagram of the algorithm for calculation of heat power consumption

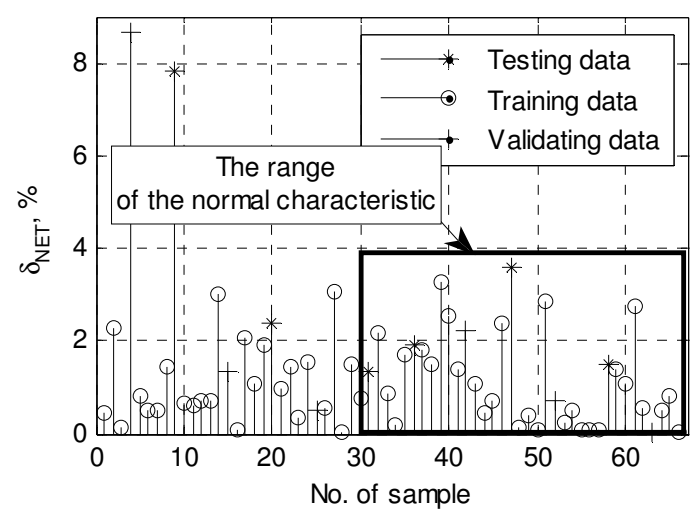

Fig. 9. Relative errors $\delta_{N E T}$ of the network with 2 neurons in the hidden layer (the first variant of input data selection)

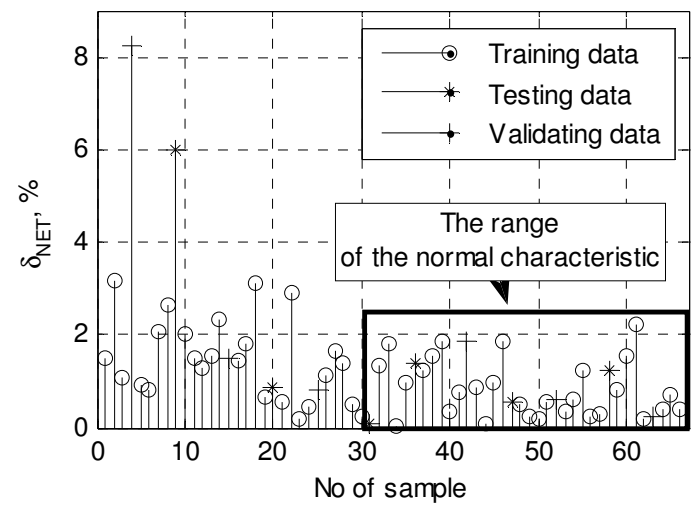

Fig. 11. Relative errors $\delta_{N E T}$ of the network with 2 neurons in the hidden layer (the second variant of input data selection)

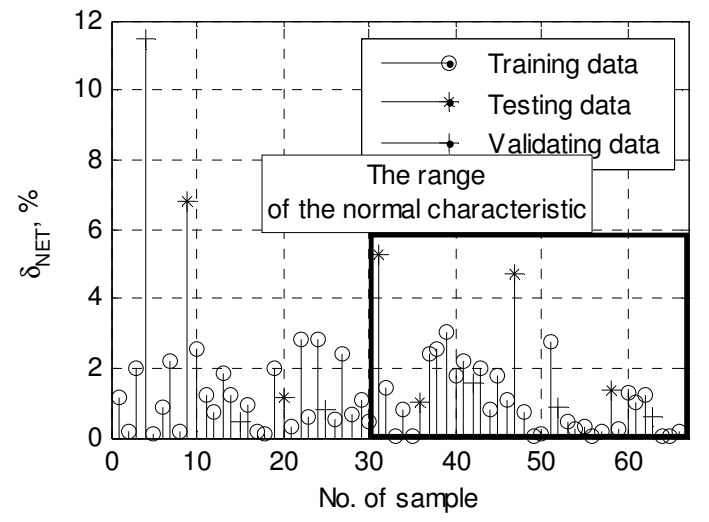

Fig. 10. Relative errors $\delta_{N E T}$ of the network with 10 neurons in the hidden layer (the first variant of input data selection)

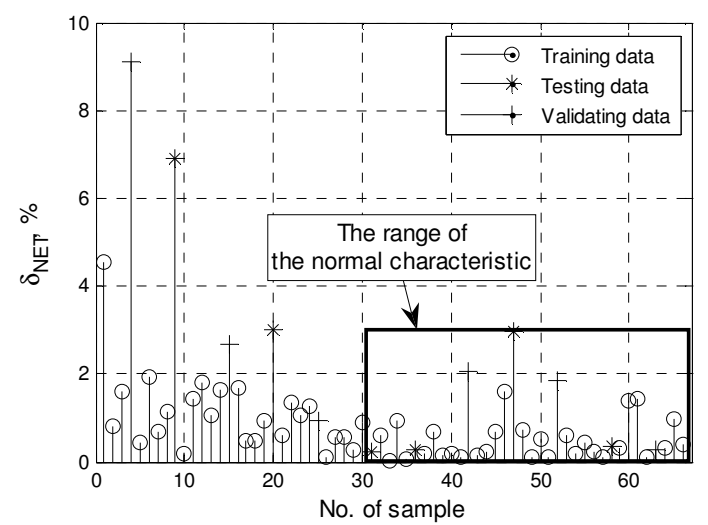

Fig. 12. Relative errors $\delta_{N E T}$ of the network with 10 neurons in the hidden layer (the second variant of input data selection) 